\title{
Estudio preliminar de paisaje del sector noroeste del municipio de Vitoria-Gasteiz
}

\author{
Barreal, S. ${ }^{1}$, Vilela, J. ${ }^{1}$, Marañón, B. ${ }^{1 *}$ \\ ${ }^{1}$ Centro de Estudios Ambientales (CEA) - Ayuntamiento de Vitoria-Gasteiz-Vitoria-Gasteizko Udala. \\ Pintor Teodoro Doublang 25, 01008, Vitoria-Gasteiz
}

El trabajo se ha realizado en el marco del Plan Comarcal de Empleo del Ayuntamiento de Vitoria-Gasteiz 2015 (iniciativa promopvida por Lambide-Servicio Vasco de Empleo).

*Autor para correspondencia: bmaranon@vitoria-gasteiz.org

\section{Resumen}

El municipio de Vitoria-Gasteiz es un territorio muy estudiado, sobre el que se dispone de gran cantidad de información; sin embargo se echa en falta un estudio que aglutine todo el conocimiento adquirido y determine las características paisajísticas del municipio a escala local. Para suplir esta carencia, y a modo de estudio piloto, se planteó realizar un estudio de paisaje en el sector noroeste del mismo con el objeto de contrastar la metodología utilizada. La elección de dicho sector responde a la diversidad de características y ambientes que comprende, así como a su representatividad en el conjunto del municipio. Tras un primer estudio de fuentes documentales, se delimitaron distintas unidades de paisaje en función de una serie de criterios determinados por cerramientos visuales, ecológicos y antrópicos. Seguidamente, mediante consulta documental y cartográfica y trabajo de campo, para cada una unidad de paisaje se elaboró un inventario, caracterización y diagnóstico de sus principales elementos paisajísticos (a través de matrices DAFO) y así como su correspondiente cartografía. De este modo, se cumple el objetivo planteado de lograr un conocimiento exhaustivo del área de estudio para poder plantear en el futuro objetivos de calidad paisajística y las pertinentes estrategias de gestión, ordenación y conservación del territorio.

Palabras clave: diagnóstico de paisaje, impactos, recursos paisajísticos, unidad de paisaje. 


\section{Introducción}

El 21 de julio de 2009 el Gobierno Vasco se adhirió al Convenio Europeo del Paisaje (Consejo de Europa, 2000) adoptando su definición de paisaje y adquiriendo como propia la obligación de cumplir sus contenidos. Este compromiso se materializó mediante la formulación del Decreto 90/2014, de 3 de junio (Gobierno Vasco, 2014), sobre protección, gestión y ordenación del paisaje en la ordenación del territorio en la Comunidad Autónoma del País Vasco. El Convenio Europeo define paisaje como «cualquier parte del territorio tal como la percibe la población, cuyo carácter sea el resultado de la acción y la interacción de factores naturales y/o humanos».

De este modo, el paisaje viene definido por la complejidad en su estructura y múltiples componentes o variables. Su definición abarca una amplia gama de elementos bióticos, abióticos, actuaciones humanas, modificaciones naturales o artificiales, incorporando las actividades antrópicas que se asientan sobre el paisaje y el uso del mismo, así como la percepción de todos estos caracteres, reflejo de los acontecimientos históricos sucedidos en ese terreno y de la propia dinámica del paisaje, susceptible de cambios, que lo ha conducido a su estado actual. Así, la importancia del paisaje alcanza relevancia en las esferas culturales, medioambientales, sociales y económicas que componen la identidad, bienestar social y patrimonial del territorio; lo que deriva en la necesidad de integración equilibrada y sostenible del paisaje y sus recursos en las distintas políticas.

\section{Objetivos}

El objetivo principal de este estudio de paisaje es adquirir un conocimiento detallado de los distintos elementos que componen el paisaje correspondiente al sector noroeste del municipio de Vitoria-Gasteiz, con el fin último de establecer las medidas y/o actuaciones apropiadas para mejorar o mantener una estructura de paisaje equilibrada que conserve y garantice parámetros de calidad mediante instrumentos de protección, gestión y ordenación del territorio y de divulgación.

Los objetivos específicos planteados son:

- Desarrollar y aplicar una metodología adecuada para la realización de un inventario y diagnóstico de paisaje a escala local.

- Aplicar los criterios necesarios para delimitar unidades de paisaje en el ámbito de estudio y efectuar un diagnóstico y una valoración de las mismas.

- Recopilar la información referente a los elementos que componen el paisaje de la zona noroeste del municipio de Vitoria-Gasteiz.

- Establecer las bases para el futuro desarrollo de un Plan de Acción del Paisaje del sector noroeste del término municipal de Vitoria-Gasteiz.

\section{Metodología}

Atendiendo a los objetivos mencionados, se plantearon las siguientes fases refle- 
jadas en la Fig. 1 para llevar a cabo el estudio de paisaje. Hasta el momento, se han llevado a cabo las dos primeras.

\section{ESTUDIO DE PAISAJE \\ DEL SECTOR NOROESTE DEL MUNICIPIO DE VITORIA-GASTEIZ}
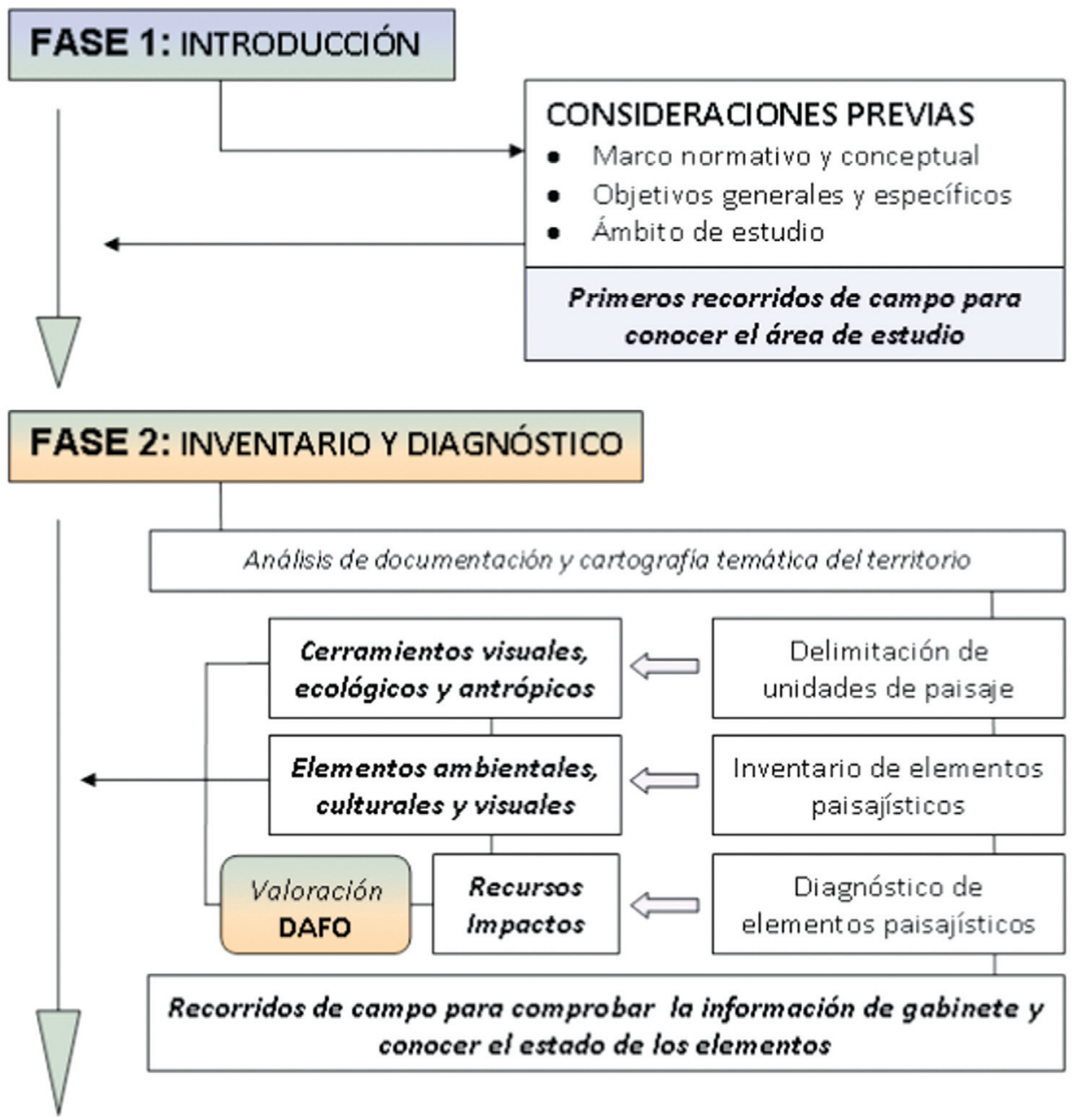

\section{FASE 3: PLAN DE ACCIÓN DEL PAISAJE}

Figura 1. Esquema del procedimiento planteado para el estudio de paisaje.

\section{1. Ámbito de estudio}

En cuanto a la organización territorial y administrativa de Álava, es preciso reseñar sus peculiaridades. Primeramente, el Territorio Histórico se divide en cuadri- 
1las (Zuia, Añana, Ayala, Campezo-Montaña Alavesa, Laguardia-Rioja Alavesa, Salvatierra y Vitoria-Gasteiz), cada una de las cuales cuenta con varios municipios. A su vez, el municipio está compuesto por las Juntas Administrativas o Concejos, pequeñas unidades territoriales con competencias propias y una gran cercanía a la población.

El municipio de Vitoria-Gasteiz, perteneciente a la Cuadrilla de Vitoria-Gasteiz, cuenta con 63 concejos, además de la propia ciudad. Estos concejos están integrados en la zona rural del término municipal de Vitoria-Gasteiz. El ámbito de estudio se centra en el sector noroeste del municipio de Vitoria-Gasteiz y comprende los siguientes 17 pueblos (Fig. 2): Antezana de Foronda, Aránguiz, Artaza, Asteguieta, Estarrona, Foronda, Guereña, Hueto Abajo, Hueto Arriba, Legarda, Lopidana, Mandojana, Martioda, Mendiguren, Mendoza, Ullíbarri-Viña, Yurre.

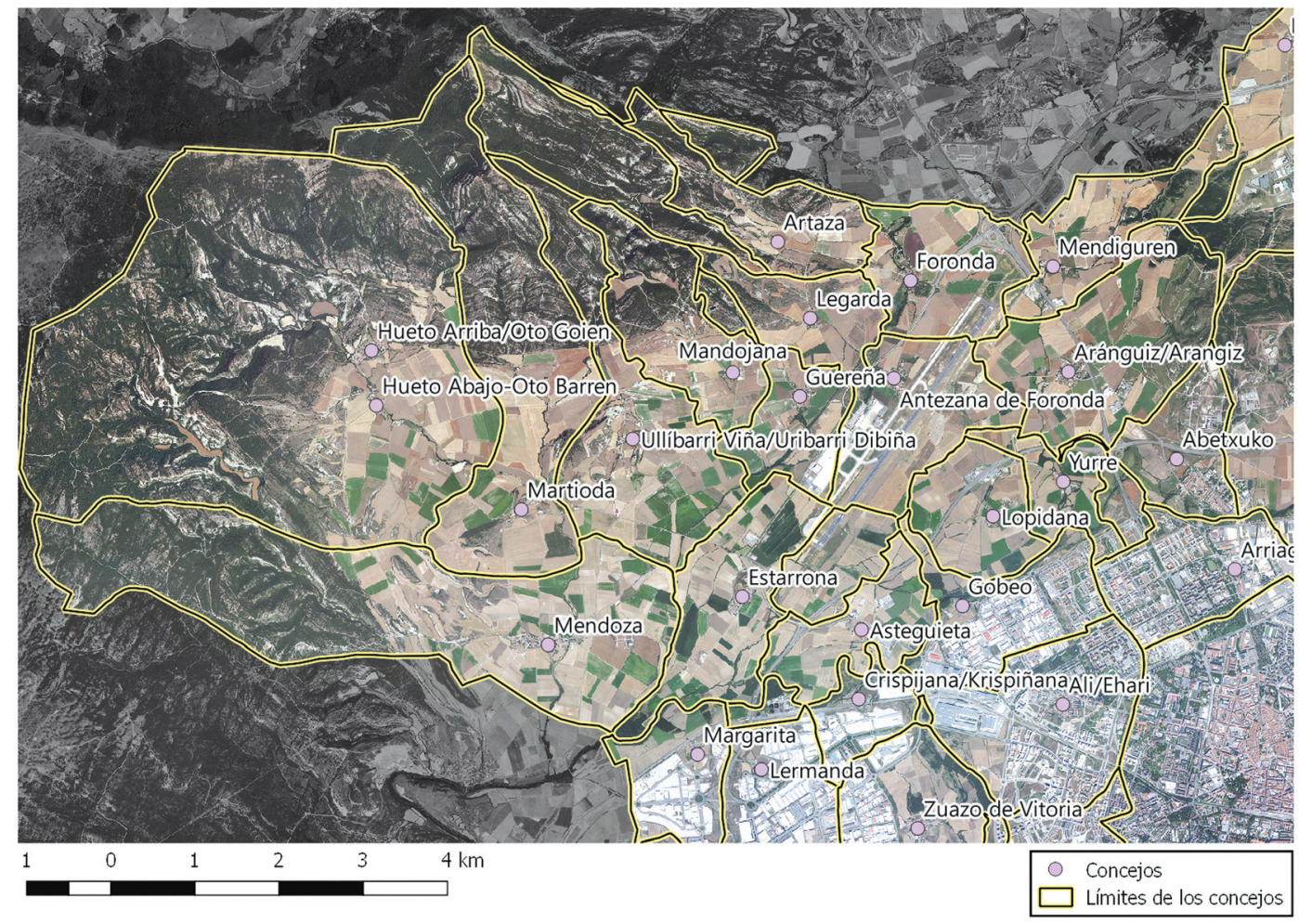

Figura 2. Mapa de los términos concejiles del sector noroeste del municipio de Vitoria-Gasteiz.

Sin embargo, el paisaje no se adscribe a los términos legales de separación, sino que se establece en un continuo físico y perceptivo, como establece la propia definición de paisaje previamente mencionada. Así, los límites estrictos del área de estudio se determinan una vez definidas todas las unidades de paisaje y sus respectivas fronteras. 


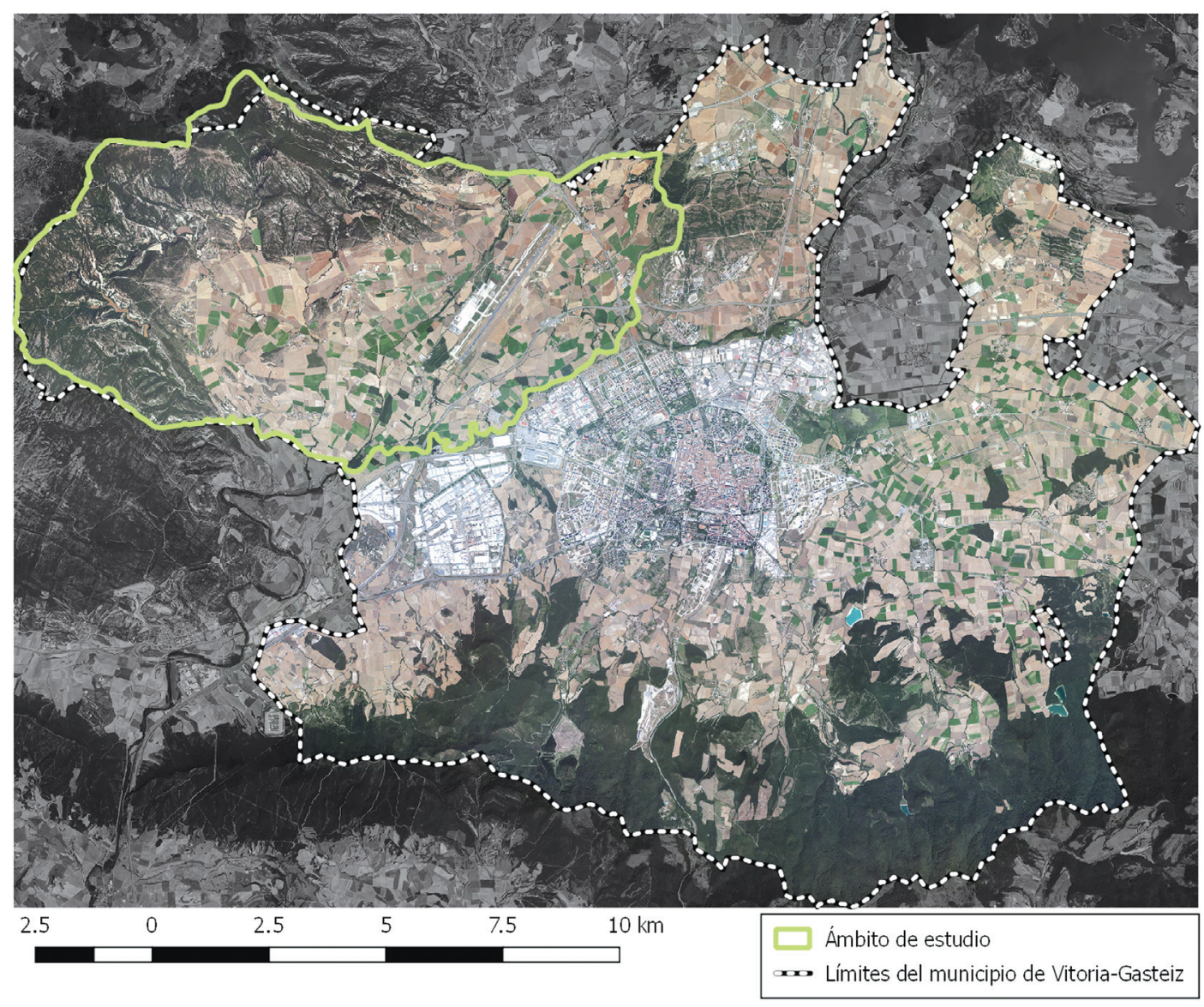

Figura 3. Mapa del ámbito de estudio.

Como se puede observar en la Fig. 3, si bien el límite administrativo y el del ámbito de estudio se encuentran muy cercanos, no coinciden completamente, ya que como es lógico el trazado del área de estudio resulta mucho más sinuoso e irregular que el del propio municipio.

Tanto en el ámbito de estudio como en el resto de la zona rural del municipio, los pueblos (entidades locales menores), sobre todo los más próximos a la ciudad sufren una relación ambivalente con respecto a la capital. Por un lado, los beneficios que aporta la proximidad de Vitoria-Gasteiz son evidentes (comunicaciones, empleo, acceso a servicios de sanidad y educación, etc.). Por otra parte, la actividad ligada al medio rural (agricultura, ganadería, forestal) ha ido disminuyendo a medida que los empleos se concentran en la ciudad, reduciendo el vínculo de los habitantes con su entorno. Esta realidad tiene un claro reflejo en forma de cambios en el territorio. En vista de todo ello, resulta necesario llevar a cabo un estudio de paisaje que ponga de manifiesto estos cambios, cómo son percibidos por la población y cuál es la mejor manera de integrarlos. 
El ámbito de estudio abarca un área de unos 75,7 km², lo que supone un 27,3\% del territorio del municipio y un 2,5\% respecto al total de la provincia de Álava. Se encuentra situado en el extremo occidental de la comarca de la Llanada Alavesa, en el centro del Territorio Histórico. Al norte limita con los municipios de Zigoitia y Zuia; al este, la Sierra Brava de Badaia linda con Kuartango y, al sur, se encuentra el municipio de Iruña de Oca. Al sureste, limita con la ciudad de Vitoria, rodeada por el Anillo Verde, representado en este tramo por el cauce fluvial y las riberas del río Zadorra. El ámbito continúa desde esta cuenca siguiendo la fisiografía del monte de Araka hasta su término al norte.

Se trata de un territorio prácticamente llano, ocupado por cultivos en su mayor parte, entre los que asientan 17 pequeños núcleos rurales. Intercalados en la zona llana, aparecen algunos cerros margosos, característicos del paisaje de la Llanada Alavesa. El sector está enmarcado al norte por las elevaciones de las sierras de Badaia y Arrato, de relieve abarrancado (barrancos de Uriño y de Oca), y al sur, por el río Zadorra. Estos límites naturales se corresponden con espacios naturales de interés para la flora y la fauna en un entorno netamente agrícola. Es necesario destacar la importancia del río Zadorra en este tramo y un reducto de robledal éutrofo (el bosque de Calzazarra), junto al mismo, declarados ambos Zonas de Especial Conservación (ZEC) dentro de la Red Natura 2000.

El territorio se encuentra fuertemente marcado por la presencia de dos grandes vías de comunicación ampliamente transitadas: la A-1 o Autovía del Norte y la N-622; así como por la presencia del Aeropuerto de Vitoria, actualmente especializado en el tráfico de mercancías. También existen algunas zonas industriales, alrededor de las dos vías de comunicación mencionadas, y algunas construcciones asociadas a la actividad agroganadera.

\subsection{Delimitación de unidades de paisaje}

La metodología para delimitar las unidades de paisaje en el área de estudio se determinó en base a la escala de trabajo del proyecto, en este caso, en torno a 1:20.000. A escala local es necesario alcanzar un gran nivel de detalle para hallar los atributos distintivos de las unidades que conforman el ámbito. Si bien es posible que algunos rasgos se compartan en varias unidades, las técnicas empleadas determinaron que la interacción entre los elementos presentes y el estado en que se encuentran alcanza la singularidad necesaria para demarcar unidades diferentes.

De este modo, los criterios empleados para delimitar las distintas unidades de paisaje se agruparon en las siguientes categorías: visuales, ecológicos y antrópicos.

En cuanto a criterios visuales, se tomaron como cerramientos visuales todos aquellos elementos que por su tamaño y/o ubicación destacan sobre el resto de elementos del paisaje, es decir, que constituyen fronteras o barreras escénicas. Para ello, se llevó a cabo un análisis de la cartografía topográfica del territorio, a partir del cual se determinaron las principales líneas visuales presentes en el área de estudio. Los cerramientos ecológicos comprenden aquellos elementos que bien por su abundancia, rareza o contraste determinan cambios en el paisaje, debidos a la vegetación, fisiografía, usos del suelo, hidrografía, etc. Para determinar estas fronteras ecológicas, se examinaron aquellas variables ambientales que determinan cambios perceptibles en el paisaje. Como 
cerramientos antrópicos, encontramos todas aquellas barreras derivadas de construcciones y actividades humanas, como industrias, vías de comunicación, infraestructuras, etc.

A continuación, se llevó a cabo la superposición de la cartografía referente a estos criterios, así como su comprobación en campo. En total, se definieron 10 unidades de paisaje para el ámbito de estudio (Tab. 1, Fig. 4).

Tabla 1. Área y pueblos presentes en cada una de las unidades de paisaje delimitadas.

\begin{tabular}{cccc}
\hline & Unidad de Paisaje & Área $\mathbf{( k m}^{\mathbf{}} \mathbf{)}$ & Pueblos \\
\hline 1 & Zadorra & 4,55 & Asteguieta, Lopidana, Yurre \\
\hline 2 & Araka & 5,39 & Aránguiz, Mendiguren \\
\hline 3 & Aeropuerto & 9,04 & Antezana de Foronda \\
\hline 4 & Estarrona & 8,73 & Estarrona, Mendoza. \\
\hline 5 & Foronda & 7,77 & Artaza, Foronda, Guereña, Legarda, Mandojana \\
\hline 6 & Cerros & 6,90 & Martioda, Ullíbarri-Viña \\
\hline 7 & Los Huetos & 7,42 & Hueto Abajo, Hueto Arriba \\
\hline 8 & Barranco de Oca & 2,77 & - \\
\hline 9 & Sierra de Arrato & 6,23 & - \\
\hline 10 & Sierra de Badaia & 16,94 & \\
\hline & Total & 75,74 & \\
\hline
\end{tabular}

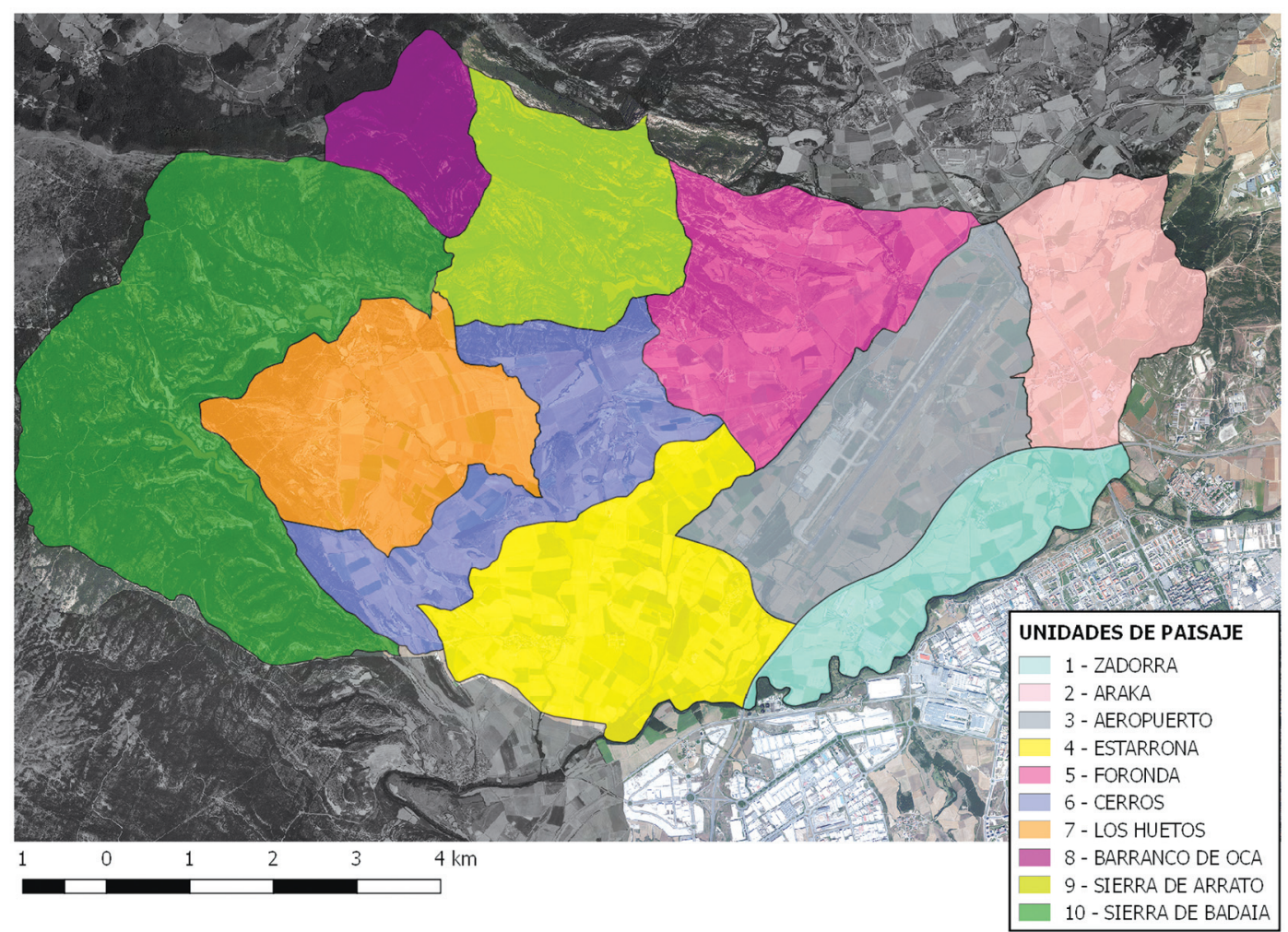

Figura 4. Mapa de las unidades de paisaje del ámbito de estudio. 


\subsection{Inventario, diagnóstico y valoración DAFO de las unidades de paisaje}

Para reflejar el estado de las unidades de paisaje del ámbito de estudio, se elaboró una ficha descriptiva para cada una de ellas, conteniendo el inventario, diagnóstico y valoración DAFO (debilidades, amenazas, fortalezas y oportunidades) de sus elementos.

En primer lugar, en cada ficha, se recogió una descripción general de la respectiva unidad de paisaje, acompañada de un mapa de la misma.

\subsubsection{Inventario de elementos que caracterizan las unidades de paisaje}

Para elaborar el inventario, se recopiló la información existente de todos aquellos elementos que caracterizan las unidades de paisaje (Tab. 2). Entendemos como elemento que caracteriza el paisaje aquel que por sus características, situación, tamaño, singularidad o funciones posee un lugar destacable en el paisaje y realiza una aportación a ese entorno por el simple hecho de encontrarse ahí. Así, para este estudio se clasificaron los elementos que caracterizan las unidades de paisaje en tres grupos principales: ambientales, culturales y visuales.

Elementos ambientales. Son elementos ambientales todas aquellas áreas o elementos bióticos y abióticos que influyen, condicionan o forman parte del medio natural.

Elementos culturales. Áreas o elementos que forman parte de la identidad local, ya sea por su construcción, antigüedad, historia, religión, servicios, patrimonio, etc.

Elementos visuales. Aquellas áreas o elementos que destacan sobre el resto y afectan a la percepción visual.

\subsubsection{Diagnóstico de los elementos: recursos e impactos paisajísticos}

Una vez listados los elementos presentes en cada unidad de paisaje, se procedió a su diagnóstico y comprobación en campo. Según su estado de conservación y afección al paisaje, los elementos se tipificaron en recursos e impactos.

Las posibles propuestas de actuación para los recursos paisajísticos tienen que ver con su protección y conservación.

Las propuestas de actuación para los impactos se dirigen a la eliminación del factor causante del impacto, a su corrección o minimización, así como a la restauración e integración en el entorno del elemento afectado.

De este modo, tras combinar el trabajo de gabinete y el diagnóstico in situ, en la ficha de cada unidad de paisaje se organizaron los elementos en estas dos categorías. Junto al diagnóstico, se incluyó la cartografía de los elementos ambientales, culturales y visuales, así como un repertorio fotográfico de los principales recursos e impactos identificados.

\subsubsection{Matrices DAFO: valoración de las unidades de paisaje}

Finalmente, se llevó a cabo un análisis DAFO para cada unidad de paisaje. Esta metodología, basada en la identificación de las principales debilidades, amenazas, fortalezas y oportunidades, facilita el establecimiento de los objetivos de calidad paisajísti- 
Tabla 2. Elementos y aspectos inventariados para la caracterización de las unidades de paisaje (UP).

\section{ELEMENTOS AMBIENTALES}

Geología y geomorfología Relieve, elementos de interés geológico o geomorfológico, cavidades o cuevas, sierras y vaguadas, cerros.

\begin{tabular}{cl}
\hline Suelos & $\begin{array}{l}\text { Erosión, usos del suelo, actividades o instalaciones potencialmente } \\
\text { contaminantes del suelo, roturos. }\end{array}$ \\
\hline Hidrología & $\begin{array}{l}\text { Cuencas hidrográficas, humedales y charcas; calidad y protección de } \\
\text { las aguas, inundabilidad. }\end{array}$ \\
\hline Vegetación & $\begin{array}{l}\text { Vegetación potencial, vegetación actual, árboles singulares, } \\
\text { vegetación de ribera, bosques isla, zonas de jardín, plantaciones, } \\
\text { frutales, catálogo de paisaje singulares y sobresalientes de la CAPV. }\end{array}$ \\
\hline $\begin{array}{c}\text { Conectividad entre Sistemas } \\
\text { Fluviales o Terrestres }\end{array}$ & $\begin{array}{l}\text { Presencia/ausencia de setos o hilera de árboles, conexión entre los } \\
\text { mismos y las cuencas hidrográficas. }\end{array}$
\end{tabular}

\section{ELEMENTOS CULTURALES}

Bienes/espacios de interés Yacimiento, edificaciones catalogadas/protegidas por el Gobierno arqueológico o arquitectónico Vasco y el PGOU (casas, palacios, torres, etc.).

Bienes inmuebles de carácter religioso

Iglesias, ermitas, cruceros, vía crucis, humilladeros.

Elementos menores Fuentes, lavaderos, abrevadero, puentes, juegos de bolos, molinos.

Accesos y conexión entre UP Vías de comunicación, caminos, puentes y rutas de acceso.

Vías verdes y zonas Itinerarios, zonas de ocio y esparcimiento.

recreativas

Elementos inmateriales Memorias, leyendas.

\section{ELEMENTOS VISUALES}

Configuración espacial del paisaje

Límites visuales e intervisibilidad entre UP

Puntos de interés visual

Tramos de interés visual

Áreas de afección visual desde ELM

y vías de comunicación

Elementos de intrusión visual
Paisaje panorámico, cerrado, focalizado o dominado.

Límites visuales establecidos según la topografía del terreno.

Visibilidad del resto de UP.

Miradores y cumbres.

Carreteras, itinerarios, caminos con setos o muros, etc.

Vistas desde puentes, localidades, carreteras y caminos.

Vallas publicitarias, carteles, pabellones, almacenes, industrias e infraestructuras, acopios, chabolas, vallados, vertidos, fincas o edificaciones en mal estado, obras, rellenos, movimientos de tierra, etc. 
ca y de las propuestas de actuación asociadas. Ello permitirá adoptar las estrategias de gestión más idóneas a fin de incrementar la calidad paisajística de este espacio.

\section{Resultados y discusión}

Dado que describir los resultados de inventario, diagnóstico y valoración de las diez unidades paisajísticas del ámbito ocuparía mucha extensión, a modo de ejemplo para conocer el método aplicado, el nivel de detalle y los resultados derivados, se muestra a continuación la información más reseñable de la ficha referente a la unidad de paisaje (UP) 1 - Zadorra.

Esta unidad, de $4,55 \mathrm{~km}^{2}$, se encuentra limitada al sur por el propio río Zadorra, y al norte por la A-1 o Autovía del Norte. Al este llega hasta el entorno del humedal de Aramatea y los límites visuales determinados por la fisiografía ascendente hacia el monte Araka. Se trata de una zona muy llana y homogénea; engloba los pueblos de Asteguieta, Lopidana y Yurre y se encuentra muy próxima a la ciudad de VitoriaGasteiz (se sitúa a escasos $5 \mathrm{~km}$ de su centro) y al Anillo Verde que la rodea.

Atendiendo a su grado de naturalidad, se decidió dividir la unidad en dos subunidades: el entorno de Asteguieta (más industrial y degradado), y el resto de la uni-

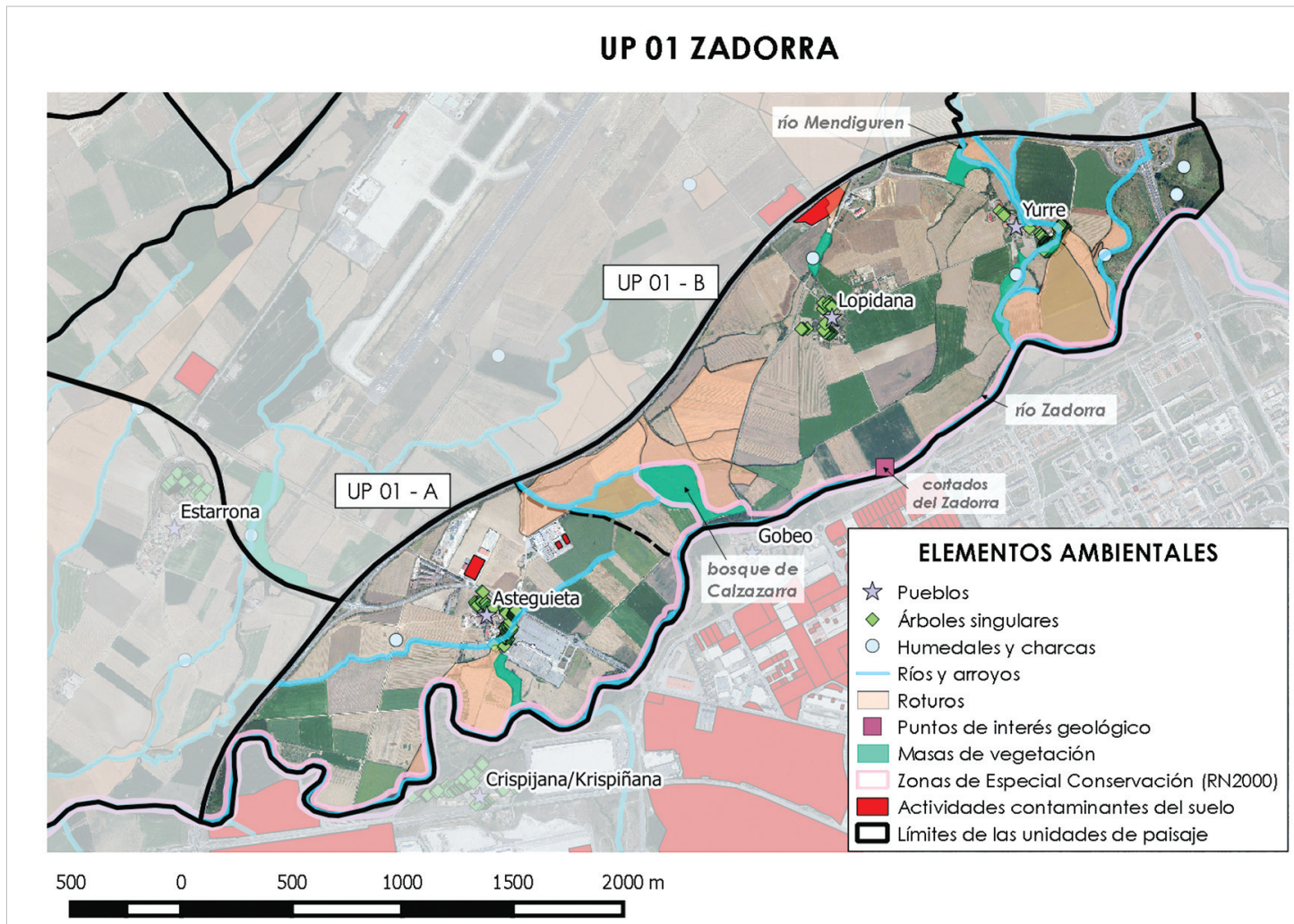

Figura 5. Mapa de elementos ambientales de la unidad de paisaje 1 - Zadorra. 
dad (de mayor interés naturalístico). Las unidades de paisaje colindantes son: 2 Araka, 3 - Aeropuerto y 4 - Estarrona.

A continuación, con la información recopilada sobre los elementos ambientales se elabora la cartografía de recursos e impactos (Fig. 5). Esta unidad posee diversas actividades o instalaciones potencialmente contaminantes del suelo y determinadas actividades que provocan daños a la vegetación y las riberas. Como recursos destacados se incluyen las dos zonas ZEC correspondientes al río Zadorra y al robledal isla de Calzazarra.

En esta unidad de paisaje, exceptuando algún impacto derivado de la presencia de la A-1, en general la mayoría de sus elementos culturales se encuentran en buen estado, por lo que fueron clasificados como recursos (Fig. 6).

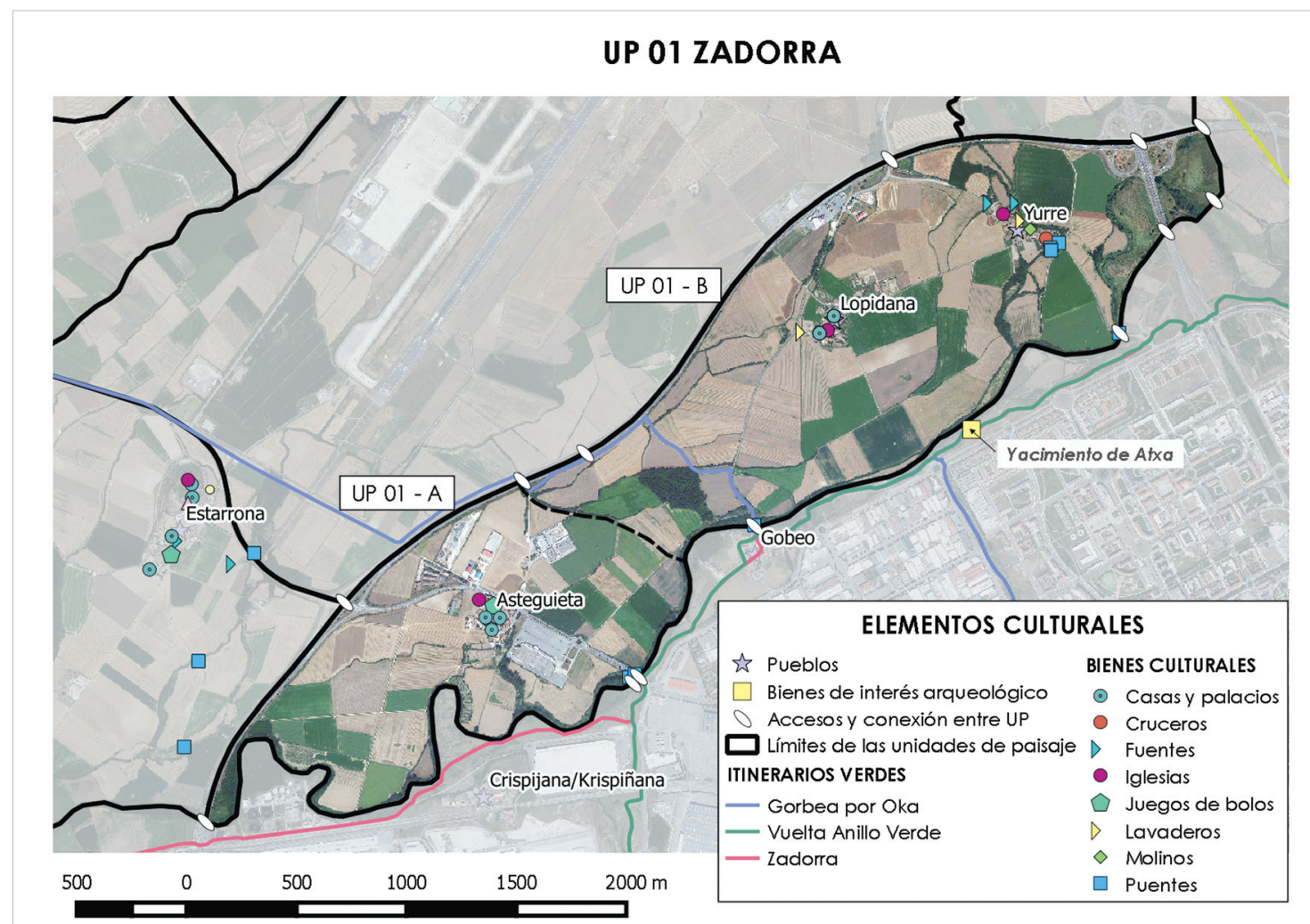

Figura 6. Mapa de elementos culturales de la unidad de paisaje 1 - Zadorra.

Visualmente, es de destacar el límite constituido por el río Zadorra. Los únicos puntos de mirador desde los cuales observar la planicie sobre la que se asienta esta unidad de paisaje se corresponden con ciertos tramos del paseo del Anillo Verde y, en concreto, el yacimiento de Atxa (Fig. 7). La mayor parte de los impactos, en forma de elementos de intrusión visual, aparecen de nuevo en el entorno de Asteguieta (Fig. 8).

Para concluir, como discusión, se muestra la matriz DAFO de la unidad de paisaje 1 - Zadorra (Tab. 3), que recoge de forma pormenorizada el análisis realizado. 


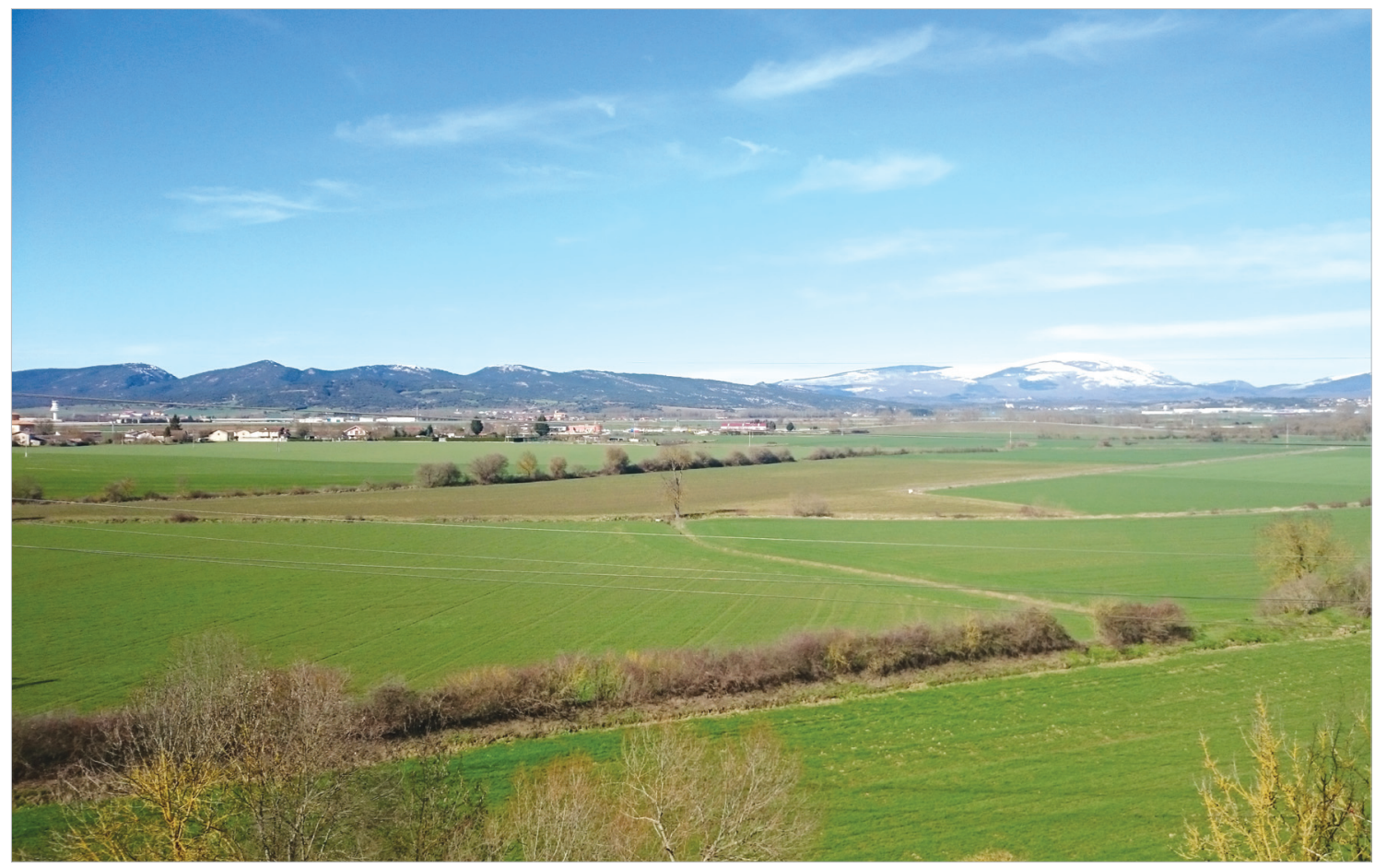

Figura 7. Panorámica de la unidad de paisaje 1 - Zadorra desde el mirador de Atxa

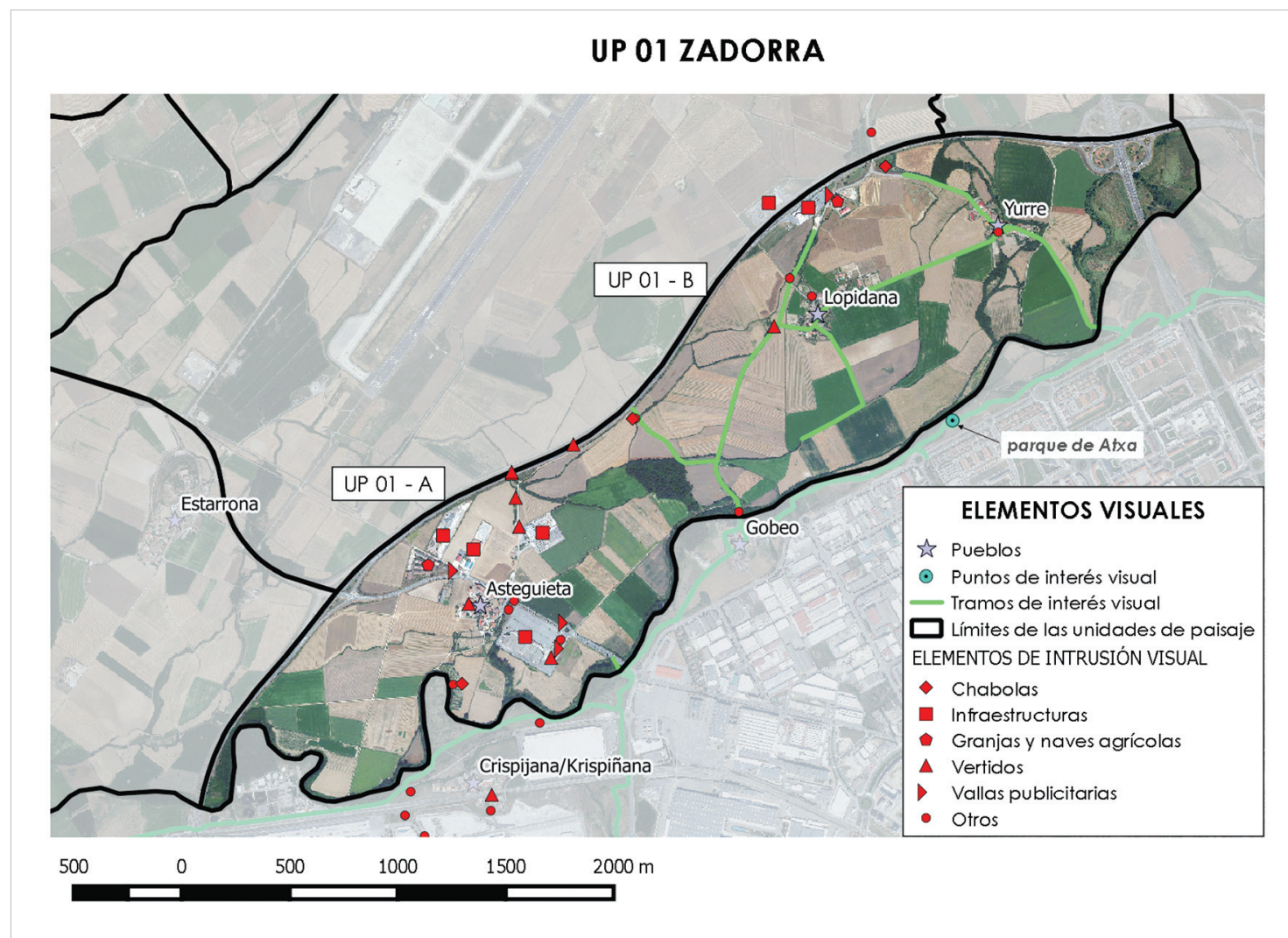

Figura 8. Mapa de elementos visuales de la unidad de paisaje 1 - Zadorra. 
Tabla 3. Matriz DAFO de la unidad de paisaje 01 Zadorra.

Tabla 3. Matiz DAFO de la unidad de paisaje 01 Zadora.

\section{MATRIZ DAFO}

\section{DEBILIDADES}

- El escaso relieve del terreno determina que cualquier infraestructura o elemento que exista o pueda existir en el futuro tenga una repercusión visual importante.

- Los abonos de los cultivos agrícolas contribuyen a mantener niveles de contaminación en el acuífero y las cuencas del Zadorra y el Mendiguren.

- La expansión de los campos de cultivo, roturos y huertas acechan la conservación de la escasa y aislada vegetación potencial de robledal existente. Conllevan además consecuencias negativas como degradación de las riberas del Zadorra y el Mendiguren, erosión de su talud, ocupación de márgenes, vertidos y ocupación de suelos, chabolas de importante incidencia visual, etc.

- No está previsto un retorno a suelo forestal de los roturos.

- En la periferia de la UP existen caminos que únicamente dan acceso a ciertas parcelas y cultivos y otros que se cortaron con la construcción de la autovía del norte.

- Las industrias e infraestructuras existentes en funcionamiento o abandonadas están clasificadas como actividades o industrias potencialmente contaminantes del suelo (Eroski, granja abandonada y desgüaces de Asteguieta, cocinas Apaolaza, restaurante y gasolinera de Lopidana, Chimeneas Inmoar y un pabellón agrícola), de gran tamaño en su mayoría, poseen un enorme impacto paisajístico en el entorno de Asteguieta y al norte de Lopidana, con impactos de magnitud asociados como vertidos, antiguas zonas de jardín abandonadas y fincas en mal estado. Además, al tratarse algunos de emplazamientos contaminantes amenazan la conservación del suelo, la hidrología y la vegetación.

- Existen áreas de vegetación degradadas o tramos con pérdida de conectividad que requieren de actuaciones, como el caso de la ribera desnuda del puente de Gobeo.

\section{FORTALEZAS}

- Se trata de un paisaje panorámico al que la combinación de cultivos y vegetación en buen estado le aportan riqueza y diversidad, así como la percepción de las montañas como fondo escénico.

- Es posible observar la UP casi de modo íntegro, si bien se distinguen dos áreas visuales correspondientes a UP 01 - A y UP 01 - B.

- La continuidad entre la vegetación asociada a las cuencas y subcuencas presentes para aumentar la conectividad ecológica entre estos sistemas y con el propio Zadorra y el Anillo Verde, los cuales establecen el límite visual de la unidad, marco cerrado por la Sierra de Badaia y el resto de cadenas montañosas. Por tanto, la mayoría de los márgenes y vegetación de los ríos Zadorra y Mendiguren se encuentran bien conservados, incluso existe conexión entre sus riberas.

- La presencia de vegetación en las cercanías de las carreteras hace función de pantalla, coincidiendo con los tramos de calidad visual.

- Esta unidad posee bastantes elementos hidrológicos con elementos de vegetación asociados de carácter destacable que, junto con los numerosos puentes repartidos por toda la UP, aportan valor al paisaje.

- Tanto el curso del Zadorra como el Robledal isla de Calzazarra, designados ZEC, paisajes sobresalientes y espacios naturales de interés, presentes en esta unidad constituyen áreas importantes para la biodiversidad y conexión ecológica municipal. La denominación de ZEC de estas áreas garantiza su conservación y protección.

- El itinerario al Gorbea por Oka, el paseo propuesto entre Lopidana y Yurre y el resto de caminos rurales en buen estado ofrecen tramos de interés visual para sus múltiples usuarios, que incluyen numerosos elementos de valor natural, especialmente accesibles, entre los cuales encontramos los cortados del Zadorra, robledal isla, conectividad entre arbolado y setos y cursos fluviales, situados en la UP 01 - B. 
Tabla 3. Matriz DAFO de la unidad de paisaje 01 Zadorra. (Cont.)

\section{MATRIZ DAFO}

\section{DEBILIDADES}

- Numerosas vallas publicitarias a la entrada y a la salida de Asteguieta acentúan el impacto visual de esta zona.

\section{FORTALEZAS}

- Multitud de accesos y conexiones (13) con las unidades UP 02 Araka, UP 03 Aeropuerto y UP 04 Estarrona.y con la ciudad y el Anillo Verde: 10 por vías de comunicación, 2 pasos de carretera (estos en la UP $01-\mathrm{A}$ ) y 1 a pie.

- Gran accesibilidad visual desde las carreteras (A1, N-622, A-3302, A-3602, A-4301) con panorámicas de calidad, además de las ofrecidas por el Anillo Verde y las parcelarias existentes entre Lopidana y Yurre (UP 01 - B), así como el importante mirador situado en el yacimiento de Atxa.

- Masas boscosas de robledales centenarios al sur de Asteguieta, al norte de Lopidana y en torno a Yurre y árboles singulares presentes en las tres localidades, en gran cantidad en Asteguieta y Yurre.

- Posee bienes de interés arquitectónico, catalogados con protección: 3 casas/palacios en Asteguieta y las iglesias presentes en los 3 pueblos. El resto de bienes inmuebles y elementos menores se encuentran en buenas condiciones. Los elementos menores y demás bienes inmuebles se encuentran en buen estado y en consonancia con el entorno rural.
- La A-1 o Autovía del Norte delimita esta UP introduciendo un grado importante de fragilidad y fragmentación ecológica, con todas las consecuencias que lleva asociado (basuras, ruido, contaminación, futuras construcciones, etc.).

- La expansión del área industrial de Ali y Júndiz y cualquier problema de contaminación derivado de estas actividades o de la depuradora de Crispijana podrá tener repercusiones en la UP.
- La frontera con el Anillo Verde y la cercanía del Parque y el yacimiento de Atxa supone muchas ventajas: tramos y puntos de interés visual desde los que observar la UP, conectividad con la vegetación del Zadorra, áreas de ocio, etc. Las labores de gestión y conservación de estos entornos pueden desembocar en beneficios para el resto de esta UP, exigiéndole a su vez mantener una buena calidad del paisaje para estar en sintonía con estos elementos.

- La cercanía a la ciudad de Vitoria-Gasteiz permite llevar a cabo actuaciones y cubrir las necesidades de la UP con mayor facilidad. 
Para todas las unidades de paisaje se llevó a cabo el mismo procedimiento, obteniendo resultados ajustados a las particularidades y especificidad de cada unidad.

\section{Conclusiones}

El sector noroeste del municipio de Vitoria-Gasteiz presenta una gran variabilidad de características que se agrupan para definir y valorar las 10 unidades de paisaje planteadas en el estudio. Como síntesis, es posible señalar que las UP 2 (Araka) y UP 3 (Aeropuerto) destacan por su elevado número de impactos $\mathrm{y}$, a su vez, ven eclipsados sus recursos por la presencia de industrias y vías de comunicación. Las UP 5 (Foronda) y UP 7 (Los Huetos) contienen la variabilidad paisajística que les confiere la presencia tanto de cultivos como el inicio de las estribaciones de la Sierra de Arrato (UP 9) y la Sierra de Badaia (UP 10), respectivamente. Las unidades de las sierras, junto con la del Barranco de Oca (UP 8) destacan sobre todo por sus recursos ambientales y visuales, quedando los culturales relegados a itinerarios o elementos inmateriales. Los cerros de Estarrona (UP 4) y los cerros de Martioda y Ullíbarri-Viña (UP 6) rompen la monotonía agrícola y son referencias visuales de interés perceptibles desde gran parte del ámbito de estudio. Y, como ya se ha señalado, el río Zadorra y el Anillo Verde (UP 1) constituyen elementos de gran valor, en el límite del sector de estudio.

La escala de trabajo y la metodología seleccionadas en este trabajo han exigido un alto nivel de detalle, para lo que fueron necesarias numerosas salidas de campo, que aumentaron la complejidad del estudio ante la cantidad de elementos que diagnosticar.

En general, los recursos hallados dotan de un elevado valor intrínseco a este territorio y las prioridades de protección o restauración se han de enfocar, por ejemplo, a potenciar aquellos elementos que se encuentran aislados, como el bosque isla (UP 1 - Zadorra) y la ermita de Urrialdo (UP 6 - Cerros). Para reducir los impactos asociados a las infraestructuras e industrias se considera fundamental establecer medidas de integración paisajística. Por otro lado, muchos de los impactos pueden resultar comunes a otras zonas rurales del municipio.

En todo caso, quedan sentadas las bases para dar los siguientes pasos de cara a la elaboración de un Plan de Acción de Paisaje: compartir el diagnóstico con la ciudadanía y otros agentes sociales, establecer los objetivos de calidad paisajística y definir las actuaciones y estrategias de gestión, ordenación y conservación del territorio y el programa de seguimiento del Plan.

\section{Bibliografía}

Ayuntamiento de Errentería, 2015. Medio ambiente y montes. Plan de Acción del Paisaje en el entorno del río Oiartzun (Errentería). Disponible en: http://ingurumena.errenteria. eus/index.php/es/tematica-ambiental/paisaje/433-plan-de-accion-del-paisaje-en-el-entorno-del-rio-oiartzun Último acceso 18 Noviembre 2016. 
Ayuntamiento de Erriberagoitia/Ribera Alta, 2015. Fichas de caracterización del paisaje de Erriberagoitia/Ribera alta y el PAP (Plan de Acción del Paisaje). Disponible en: http://erriberagoitia.com/wp-content/uploads/2015/06/PDF-completo_04_06_20151.pdf. Último acceso 18 Noviembre 2016.

Ayuntamiento de Vitoria-Gasteiz, 2104. Estrategia para la conservación de la biodiversidad del municipio de Vitoria-Gasteiz. Disponible en: http://www.vitoria-gasteiz.org/ wb021/http/contenidosEstaticos/adjuntos/eu/00/33/50033.pdf Último acceso 18 Noviembre 2016.

Consejo de Europa, 2010. Convenio Europeo del Paisaje, 2010. Disponible en: http://www. magrama.gob.es/en/desarrollo-rural/temas/desarrollo-territorial/ 090471228005d489_t cm11-24940.pdf Último acceso 27 Abril 2016.

Generalitat Valenciana, consellería de infraestructuras, territorio y medio ambiente, 2012. Guía metodológica. Estudios de paisaje. Disponible en: http://www.upv.es/contenidos/ CAMUNISO/info/U0670136.pdf Último acceso 18 Noviembre 2016.

Gobierno Vasco, 2014. Decreto 90/2014, de 3 de junio, sobre protección, gestión y ordenación del paisaje en la ordenación del territorio de la Comunidad Autónoma del País Vasco.Disponible en: https://www.euskadi.eus/r48-bopv2/es/bopv2/datos/2014/06/ 1402623a.pdf Último acceso 27 Abril 2016. 